\title{
Assessing Pattern of Immunization Uptake among Inhabitants of Two Metropolitan Communities in Lagos State, Nigeria
}

\author{
Isibor I and Bassey BE* \\ World Health Organization (WHO), Nigeria Country Office, Nigeria
}

*Corresponding author: Bassey Enya Bassey, World Health Organization (WHO), Nigeria Country Office, UN House, Plot 617/618, Diplomatic Drive, Central Business District, PMB 2861, Garki, Niegeria, E-mail: bassey69@yahoo.com

\section{Research Article}

Volume 2 Issue 1

Received Date: August 30, 2017

Published Date: September 13, 2017

\section{Abstract}

Introduction: Vaccines remain one of the most successful and cost-effective public health interventions ever made. Studies have shown that the global use of vaccines saves over 2-3 million lives each year. It also prevents untold suffering and lifetime disability in millions of people.

Methods: A structure questionnaire used in data collection included questions on state of origin, exposure the oral polio vaccine from routine immunization and the last 2 rounds of Supplemental Immunization Activities (SIAs) as well as reasons for absence of immunization where found. In addition, the child's routine vaccination status was obtained from the vaccination card (where available) or by maternal history

Results: Twenty-four (24) percent of the children surveyed were within the routine immunization age range of 0-11 months. Another $23 \%$ and $15 \%$ were those aged $48-60$ and 36-47 months old respectively. High zero dose children in RI (37\%) and SIA (27\%) indicating weak immunization coverage were recorded. The overall uptake of immunization among children who received $\geq 3$ doses of vaccine is $50 \%$ and $32 \%$ for routine and supplemental immunization respectively. In all, $46 \%$ of Routine Immunization (RI) and $18 \%$ of SIA respondents did not receive the vaccine due to religious reasons

Conclusion: Routine and supplemental immunization utilization in these communities is suboptimal, religious, child absent and no felt need were the major reasons identified for the suboptimal performance.

Keywords: Immunization; Non-Compliance; Coverage

\section{Introduction}

Vaccines remain one of the most successful and costeffective public health interventions ever made. Studies have shown that the global use of vaccines saves over 2-3 million lives each year. It also prevents untold suffering and lifetime disability in millions of people [1]. However, this only happens when potent vaccine gets to the children involved. A couple of studies have shown that about 21.8 million infants each year fail to receive this life-saving vaccine [2]. The result of this is an estimated 1.5 million avoidable deaths that occur annually from vaccine-preventable diseases amongst children less than 5 years old particularly, in developing countries [2]. The World Health Organization (WHO) estimates that the 


\section{Vaccines \& Vaccination Open Access}

most culpable countries towards this are India, Nigeria and Pakistan.

Significant progress was made by Nigeria to improve immunization coverage in the 1980-90s[3]. But, most of the success achieved proved difficult to maintain resulting in a gradual decline of immunization coverage over time [3]. It has however been observed that there has been some improvements in country's immunization coverage in recent years. This is as a result of concerted efforts by both the Government and stakeholders involved in this area. The rejuvenation and strengthening of the immunization system and the acceleration of the polio eradication initiative served to drive much of this effort. To this end, the country adopted the following strategies: the Reaching Every Ward (REW) strategy, the Integrated Maternal, Neonatal and child Health (MNCH) strategy, Local Immunization days, Supplemental immunization programs such as Polio Immunization Plus Days (IPDs), accelerated measles campaign, maternal and neonatal tetanus elimination campaign, yellow fever campaigns amongst others, as a multi-pronged approach to reach the unreached.

The synergy of these strategies has presented opportunities to improve access and coverage of immunization in many communities that hitherto have fallen below the radar of immunization services. This has reduced morbidity and mortality from vaccine preventable diseases and has moved the country closer to meeting its Millennium Development Goals (MDG) [4].

In-spite of these efforts, it was recorded that about $40 \%$ of the 1 million childhood deaths that occurred in 2012, were from vaccine preventable diseases. This implies that despite these laudable strategies, relevant vaccines still fail to reach eligible recipients. A review of the progress of the MDGs in 2012 showed that MDG 4 had recorded a $52 \%$ decrease in childhood mortality from 191/1000 in 1999 live births to 94/1000 live births in 2012 [4]. Although immunization failure alone does not account for all death in children, it is known to contribute immensely.

As part of the Multiyear plan of EPI, the country plans to achieve a projected coverage rate of $87 \%$, a huge task for many states in the country.

Lagos State is the commercial nerve centre of the Nigerian economy. Although the smallest state in the country, it has the highest population with a population growth rate of about $8 \%$. This is almost twice that of the whole country. The United Nations (UN) predicts that Lagos will be the third largest megacity in the world behind Tokyo (Japan) and Bombay (India) by 2015, given its current growth rate [5]. With a population growth of about 600,000 persons per annum, the seemingly unending population dynamics in the state comes with its challenges to the health system. The state has seen a gradual improvement in routine and Supplemental Immunization Activities (SIAs) coverage of the oral polio vaccine (OPV) in the last few years. Routine coverage for oral polio vaccine 3 increased from 60.9 in 2008 to $65.0 \%$ in 2013 [3]. However, this coverage proves unstable due to the high population flow and the high birth cohort.

This paper examines the polio coverage in the two most populated Local Government areas (LGA) in Lagos state-Ajeromi Ifelodun and Lagos mainland LGAs with focus on routine and supplemental immunization coverage in some high risk communities. It also investigates the reasons for partial or non-immunization of the eligible indigenes in the communities within the LGAs.

\section{Methods}

This study represents the results of a community household survey conducted in the state in response to the detection of zero dose children in the state. A cross sectional household community survey was conducted in January and March 2015 in two LGAs in Lagos state namely: Ajeromi Ifelodun and Lagos Mainland. The survey was conducted in two wards (Alayabiagba and Aiyetoro) and 6 settlements in Ajeromi Ifelodun LGA. One ward (Otto) and one settlement were used for the survey in Lagos Mainland LGA. The wards and settlements surveyed were based on a 500 meter proximity to the zero dose case.

\section{Study location}

Ajeromi Ifelodun LGA is located in the Badagry administrative division and was created in 1996 from the old Apapa LGA and the densest LGA in the state with a population of 1,435,295and population density of 103,258.63. It covers a land mass of 13.9 sq. meters. The LGA plays host to a strongly diverse population from within and outside the country. This LGA comprises the two biggest slums of the state namely: Ajegunle and Amukoko. It is generally one of the relatively low-income, high traffic areas of the city. 


\section{Vaccines \& Vaccination Open Access}

Lagos Mainland is a one of the oldest LGAs in Lagos created in 1976 from the old Lagos city council. It has a population of 629,469 and population density of 32,083.03. It covers a landmass of 19.62. The LGA is made of two major communities-Ebute metta and Yaba. The LGA host both low and middle-income areas.

\section{Study Population}

The study population was made of mothers with children 0-59 months residing in the community at the time of the study. In order to improve community compliance with the study teams, sensitization of community leaders and gatekeepers was conducted prior to the actual survey day.

All mothers with children 0-59 months residing in the communities at the time of the survey were interviewed.

\section{Data Collection}

The survey tool was a standardized structured questionnaire used for conducting community surveys as part of detailed investigation of polio vaccine naive (zerodose) children detected as part of the polio eradication program. The tool was interviewer administered and survey teams included Expanded Programme on Immunization (EPI) staff from the state and LGA levels as well as WHO. This team also included interpreters to facilitate the communication with respondents.

The questionnaire included questions on state of origin, exposure the oral polio vaccine from routine immunization and the last 2 rounds of SIAs as well as reasons for absence of immunization where found. In addition, the child's routine vaccination status was obtained from the vaccination card (where available) or by maternal history.

\section{Results}

We administered questionnaire to mothers and caregivers of 225 children from households surveyed in two metropolitan LGAs. Table 1 shows characteristics of children surveyed. Twenty-four (24) percent of the children surveyed were within the routine immunization age range of $0-11$ months. Another $23 \%$ and $15 \%$ were those aged 48-60 and 36-47 months old respectively. We also assessed the geopolitical zone of origin of the respondents. In total, 39\% are indigenes of North West, closely by North Central with $24 \%$, while the South East recorded 3\% (Table 1).

\begin{tabular}{|c|c|c|}
\hline Age (months) & Number & Percent \\
\hline $0-11$ & 54 & 24 \\
\hline $12-23$ & 45 & 20 \\
\hline $24-35$ & 41 & 18 \\
\hline $36-47$ & 34 & 15 \\
\hline $48-60$ & 52 & 23 \\
\hline \multicolumn{2}{|c|}{ Geopolitical distribution } \\
\hline North Central & 54 & 24 \\
\hline North East & 11 & 5 \\
\hline North West & 88 & 39 \\
\hline South East & 7 & 3 \\
\hline South South & 14 & 6 \\
\hline South West & 41 & 18 \\
\hline Others & 11 & 5 \\
\hline
\end{tabular}

Table 1: Age and demographic characteristics of respondents $(\mathrm{n}=225)$.

Routine and supplemental immunization status of children surveyed was also assessed as illustrated in Figure 1. We recorded high zero dose children in RI (37\%) and SIA $(27 \%)$ indicating weak immunization coverage. The overall uptake of immunization among children who received $\geq 3$ doses of vaccine is $50 \%$ and $32 \%$ for routine and supplemental immunization respectively.

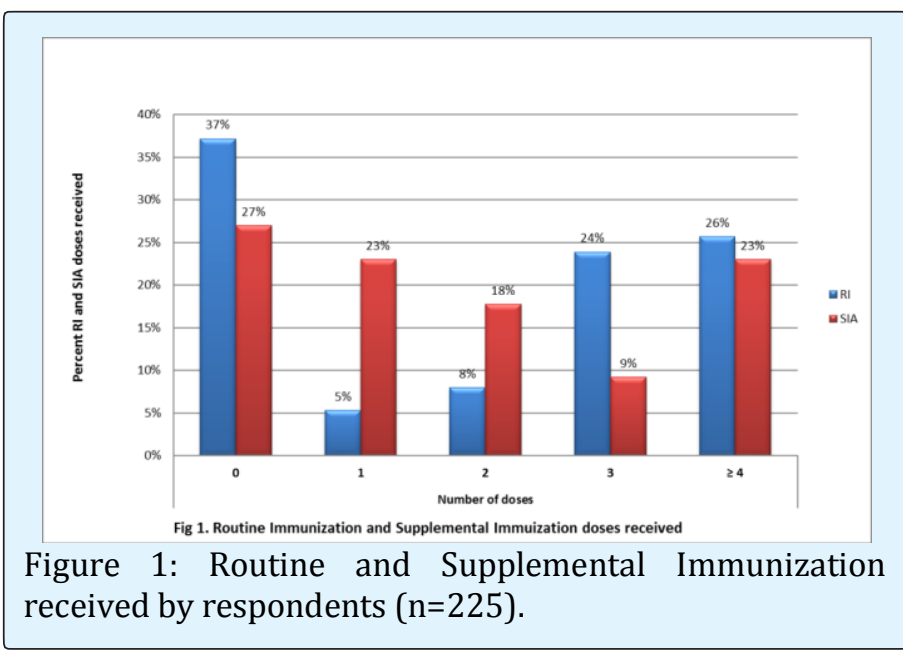

Figure 2 illustrate the reasons given by parents/caregivers for not accepting or receiving vaccines. In all, $46 \%$ of RI and $18 \%$ of SIA respondents did not receive the vaccine due to religious reasons. Also $18 \%$ said there was no felt need, while services were not provided to $17 \%$ of SIA respondents and another $13 \%$ was due to child absent. 


\section{Vaccines \& Vaccination Open Access}

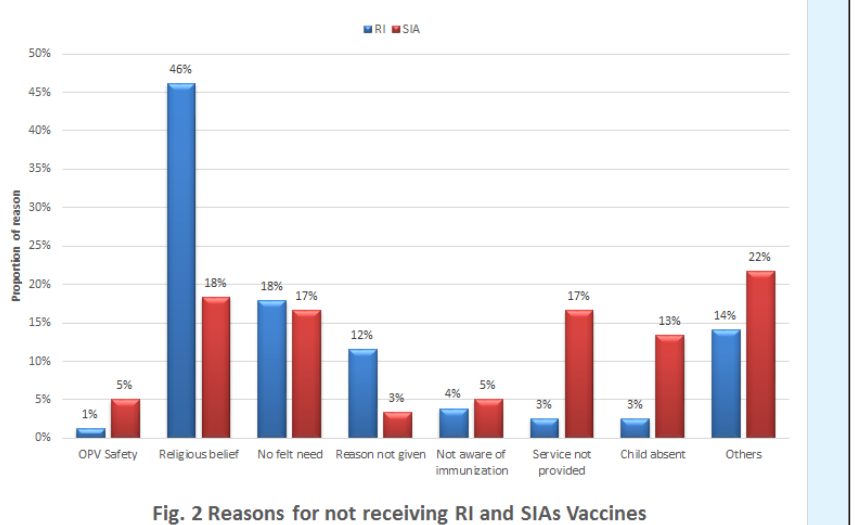

Figure 2: Reasons for not receiving routine and supplemental vaccinations $(n=225)$.

\section{Discussion}

Nigeria is closer than ever to reaching the goal of polio eradication. The country for the first time recorded the absence of the wild polio cases for over a period of 12 months. As the country crosses this significant milestone, the gold standard will be on sustaining and building on the gains achieved so far not just for polio, but also for the eradication and elimination of other diseases.

Attaining and maintaining high immunization coverage levels is the hallmark of a strong routine and supplementary immunization programme. The synergy of routine immunization and supplementary immunization activities have contributed immensely to putting Nigeria on the current trajectory and strengthening health systems.

Over the past five years, concerted efforts have been made to reverse the dismal RI performance particularly in light of achieving the MDG (particularly MDG goal 4) and actualizing the goal of polio eradication. Recent reforms in Routine Immunization systems has witnessed an improved vaccine supplies and logistics including vaccine security, distribution and safety; increase in the number of fixed and outreach session to improve access and utilization; and better session monitoring for enhanced accountability. Currently, with regards to polio, a fully immunized child should have received at least 3-4 doses of the routine polio vaccine during the first year of life and over a course of 3-4 visits including doses given at birth.

In spite of these efforts, our study revealed a sizable number of children remained unprotected from poliomyelitis with $37 \%$ having never received the routine polio vaccine. This can be linked to the ethnic heterogeneity of the study population with majority of the respondents being from the northern part of the country known for low immunization rates. In a study with a comparable heterogeneous population, Onyefara, et al. found similar ethnic disparities in coverage rates [6]. This resonates with the fact that, that part of the country is known to have one of the lowest immunization rates [3]. Studies on immunization coverage conducted among local populations in northern part of the country have generally found comparatively lower coverage rates particularly when compared to similar studies in the southern part of the country [7]. The 2013 National Demographic Household Survey conducted in the country corroborates these findings [8].

For over 10 years, the drive for a polio free Nigeria has been the top priority of both the Government and its respective partners in health World Health Organization, United Nations Children Fund). The country remained the only polio endemic country in Africa and the sub-region and one of the last 3 in the world along with Pakistan and Afghanistan [9]. Concerted efforts to redress the situation saw the frequent conduct of polio SIAs in the country particularly in the highly polio endemic northern states. During these campaigns the polio vaccine is offered to children 0-5 years of age irrespective of previous immunization status. In these states on the average, polio mass immunization campaigns are conducted at least 5-6 times annually particularly the north western and north eastern parts of the country with the highest endemicity, while the less endemic southern states generally conducted bi-annual campaigns.

In addition, the vaccine is often offered as part of other health interventions conducted in the country. This translates to several opportunities at immunization in the course of a year. It is therefore expected that an average child living in the country should have been exposed to the SIA dose of the polio vaccine at least twice during the course of a year and at least 10 times before their 5th birthday. The study however recorded low polio SIA immunization coverage rate, as $27 \%$ of the recipients had never received OPV through the SIAs. A further review of the results also revealed that a considerable number of children remain under-immunized $(22 \%)$ for polio while about a little less a quarter of the children $(17 \%)$ had received neither the routine nor supplemental polio doses.

Clearly, there are children who continue to fall below the radar of the routine and supplemental immunization 


\section{Vaccines \& Vaccination Open Access}

services leaving them vaccine naive to poliomyelitis. Understanding the reasons for un-/under-immunized will be critical to sustaining the milestones so far achieved in the eradication of polio at this time.

With regards to routine immunization services, the reasons are multi-faceted involving the individual, the family and the community. Studies have shown that reasons for under-immunization often border on issues around the actual vaccination services including inadequate knowledge on how, where, when to receive immunization and its benefits while, non-immunization has been attributed to factors related to paternal knowledge and attitudes i.e. concerns and suspicions often deeply rooted in religious, cultural social and even political beliefs, However, Babalola, et al. [10] believes that much of the reasons for these are still poorly understood and closely related to the level of social development, population densities, economic, religious, cultural and political milieu in which the individual resides. The Nigerian situation echoes much of this assumption [10].

Polio supplemental mass campaigns have been a recurring activity in the country for the last 15 years. However, the acceptance of the these SIAs have charted a delicate course in the country from the well-publicized polio vaccines boycotts in $2003 / 2004$ by five northern states (fueled by rumors about the vaccine with fears of HIV, cancer, sterility with strong religious undertone) to the harassment and killing of polio vaccination teams in the North eastern part of the country [11]. These were costly setbacks for the program that impacted heavily on the acceptance of the vaccine particularly among northern Muslims. The national and international response to these setbacks was remarkable and helped bring and keep the program on track. Strong and persistent engagement of communities' particularly traditional and religious hierarchy to engender continued public acceptance of the vaccine has been beneficial to the success of the program.

However, field experience continues to show pockets of silent refusals and non-acceptance based on religious, cultural and other reasons. The considerable number of respondents who gave religious or no reasons at all for their non-participation in SIA could actually represent groups of persons who continue to be wary of the vaccine based on historic events.

\section{Conclusion}

Routine and supplemental immunization uptake in these communities in Lagos state is suboptimal. The dynamics of population movement into the state could cause a buildup of pockets of susceptible individuals that could be detrimental to the success of RI coverage made so far. There is therefore a need to constantly monitor the entry of persons into the various communities of the state. This should be followed by a periodical internally organized medium scale campaign educating indigenes of the communities on the importance of RI while allaying any fears or doubts they may have. The use of community structure and community informants with incentives will be useful to this strategy. As the country counts down to the final eradication in the polio end-game strategy, innovative strategies that keep the program on track must be employed and deployed to reach this goal. It is therefore important that the findings of this study add to the body of information required to keep the task of polio eradication on track in the country.

\section{What is already known on this topic}

- High routine/supplemental coverage results on good herd or population immunity.

- Children under 5 years of age are the most vulnerable to vaccine preventable diseases.

\section{What this study adds}

- Although Government and partners provides opportunities for immunization services parents and caregivers underutilize these opportunities due noncompliance or refusal to accept the vaccine.

- Suboptimal immunization coverage was seen in children surveyed

Competing interests: The authors declare no competing interests.

Author's contributions: Authors were involved in community survey, data collection, analysis and manuscript drafting '

Acknowledgments: We thank: Lagos State Government and the World Health Organization Nigeria.

\section{References}

1. Andre FE, Booy R, Bock HL, Clemens J, Datta SK, et al. (2008) Vaccination greatly reduces disease, disability, death and inequity worldwide. Bulletin of the World Health Organization 86(2): 81-160. 


\section{Vaccines \& Vaccination Open Access}

2. Adeyinka D, Oladimeji O, Adeyinka F, Aimakhu C (2008) Uptake of Childhood Immunization Among Mothers Of Under-Five In Southwestern Nigeria. The Internet Journal of Epidemiology 7(2).

3. Ophori EA, Tula MY, Azih AV, Okojie R, Ikpo PE (2014) Current Trends of Immunization in Nigeria: Prospect and Challenges. Trop Med Health 42(2): 6775.

4. (2013) Nigeria MDGs Report.

5. (2014) United Nations, Department of Economic and Social Affairs, Population Division. World Urbanization Prospects: The 2014 Revision, Highlights (ST/ESA/SER.A/352).

6. Oyefara J (2014) Mother's characteristics and immunisation status of under-five children in Ojo Local Government Area, Lagos state, Nigeria. SAGE Open 4(3): 1-10.
7. Adegboye O, Kotze D, Adegboye 0 (2014) Multi-year trend analysis of childhood immunization uptake and coverage in Nigeria. J Biosoc Sci 46(2): 225-239.

8. National Population Commission (NPC) [Nigeria] and ICF International. 2014. Nigeria Demographic and Health Survey 2013. Abuja, Nigeria, and Rockville, Maryland, USA: NPC and ICF International.

9. Morales M, Tangermann RH, Wassilak SGF (2016) Progress toward polio eradication-worldwide, 2015-2016. MMWR 65(18): 470-473.

10. Babalola S (2011) Maternal reasons for nonimmunisation and partial immunisation in northern Nigeria. J Paediatr Child Health 47(5): 276-281.

11. Ghinia I, Willott C, Dadari I, Larson H (2013) Listening to the rumors: what northern Nigeria polio vaccine boycott can tell us 10 years on. Glob Public Health 8(10): 1138-1150. 